\title{
Using verbal and blind-walking distance estimates to investigate the two visual systems hypothesis
}

\author{
JEFFREY ANDRE and SHEENA ROGERS \\ James Madison University, Harrisonburg, Virginia
}

\begin{abstract}
Verbal estimates of egocentric distance are usually too short at distances greater than several meters, yet blindfolded walking to previously viewed targets can be surprisingly accurate. We present evidence from three experiments for the existence of two visual pathways with different encodings of perceived egocentric distance. We found that (1) blind-walking estimates of egocentric distance are consistently more accurate than verbal reports; (2) an indoor versus outdoor environment selectively influences verbal reports; and (3) wearing prism lenses, which displace the visual field vertically, selectively influences the blind-walking estimates. We interpret these results in terms of the two visual systems hypothesis.
\end{abstract}

We present evidence from three experiments for the existence of two visual pathways with different encodings of perceived egocentric distance. The origin of the two visual pathways hypothesis can be traced back to the late 1960s, when four researchers presented their findings at an Eastern Psychological Association meeting. At this meeting, Held (1968), Ingle (1967), Schneider (1967), and Trevarthen (1968) presented evidence from different animal species that supported the notion of separate "what" and "where" visual pathways. Trevarthen labeled one of these systems the "focal" system, which assesses fine detail within very localized spaces, and the other the "ambient" system, which assesses the larger general spaces surrounding an individual. See Leibowitz and Post (1982) for more information about the focal versus ambient distinction.

Since the introduction of this hypothesis, a number of related issues and debates have arisen. One is the cortical versus subcortical two visual system distinction. Schneider (1967) demonstrated the importance of both cortical and subcortical visual processing when he ablated either the superior colliculus (midbrain structure) or the visual cortex (cortical structure) in Syrian hamsters and found localization and pattern identification deficits, respectively.

We thank Jack Loomis, John Philbeck, and an anonymous reviewer for their insightful comments on an earlier version of this article. We also thank the administration and staff of JMU's University Recreation Center (UREC) for the use of the gymnasiums, and the following JMU undergraduate students for their help in data collection: Rebecca Brown, Thomas Quist, Rutvi Bhatt, Tyler Burton, Meghan Carty, Caitlin Corcoran, Morgan Dowell, Joey Dunsmoor, Steve Gilpin, Jennifer Gray, Stephen Quaye, Lena Rose, Dave Rosner, and Marissa Waite. Portions of these experimental findings were presented at the annual meeting of the Vision Sciences Society, 2002, Sarasota, FL; the International Conference on Perception and Action, 2003, Gold Coast, Australia; and the annual meeting of the Psychonomic Society, 2003, Vancouver, BC. Correspondence should be addressed to J. Andre, Department of Psychology, James Madison University, MSC 7401, Harrisonburg, VA 22807 (e-mail: andrejt@jmu.edu).
Other researchers, such as Ungerleider and Mishkin (1982), have shown a similar distinction within the visual cortex itself by identifying separate dorsal and ventral processing streams. Thus, another debate arose, centered on the specific function of each of these cortical streams. Ungerleider and Mishkin proposed the functional definition of a "what is it" ventral stream, which terminates in the inferior temporal lobe, and a "where is it" dorsal stream, which terminates in the posterior parietal lobe. More recently, Goodale and Milner (1992) proposed a what versus how distinction for these cortical streams. Their theory incorporates the action goal of the observer: How is the observer using the "where" information to control visually guided action? See Creem and Proffitt (2001) for a more in-depth review of the "where" versus "how" dorsalstream debate.

Although it is generally accepted that multiple visual pathways subserving different visual functions exist, questions still remain about how an individual utilizes information from these systems and what type of representations are created. In an article designed to validate the use of blindfolded walking as a way to assess perceived egocentric distance, Philbeck and Loomis (1997) have argued that a single underlying representation is responsible for perception, whether a participant is asked to generate a verbal estimate or walk blindfolded to the previously seen target. It is well established that verbal estimates tend to underestimate distances longer than 2 or $3 \mathrm{~m}$ (Cutting \& Vishton, 1995; Kelly, Loomis, \& Beall, 2004; Loomis, Klatzky, Philbeck, \& Golledge, 1998; Sedgwick, 1986; Toye, 1986; Wagner, 1985), yet blind-walking is surprisingly accurate (Loomis, Da Silva, Fujita, \& Fukusima, 1992; Thomson, 1983). It may be that neither indicator provides a precise estimate of the underlying single variable. The verbal report is assumed by some to be an accurate report of an inaccurate perceptual representation, whereas others consider verbal reports to be subject to a variety of cognitive influences (e.g., expectations, inferences, or explicit reasoning), leading to an inaccurate 
report of a possibly quite accurate representation. Foley (1977) and Philbeck and Loomis (1997) package these influences together in a hypothesized "output transformation." Such output transformations may have no effect or a different effect on the blind-walking task, leading to the observed difference in the two indicators. However, if experimental factors influence a common, underlying psychological variable, the estimates produced ought to covary quite closely.

Philbeck and Loomis (1997) conducted an experiment on the differences between verbal reports and blindwalking estimates of egocentric distance under full- and reduced-cue viewing conditions. They found the hypothesized covariance between the two dependent variables and argued for a model of space perception in which a single representation of egocentric distance is achieved on the basis of stimulus inputs. This representation is then transformed independently by the task requirements of walking behavior and by verbal report of distance. Their findings agree with those of Foley (1977), which also support a single underlying representation, in this case assessed by two separate verbal and walking estimation devices.

Alternatively, other researchers, such as Milner and Goodale (1995), argue for multiple representations that separately subserve conscious perceptual awareness of one's surroundings and egocentric localization linked to action-based tasks. This view is partly based on their study of a visual-form agnosia patient (D.F.) who could properly orient a card and place it in a mail slot but had great difficulty at a number of tasks that required a perceptual report of the orientation of the slot, including verbally describing the orientation needed for successful completion of the task, drawing a line at the appropriate orientation, matching her hand orientation to the orientation of the slot, and rotating a handheld card to match the orientation without attempting to post it (Goodale \& Milner, 1992; Milner \& Goodale, 1995). Philbeck and Loomis (1997) describe a modification to their model that could account for these findings. In this model, the internal variable "perceived distance" is still responsible for performance of both tasks, but the alternative model includes an additional internal variable controlled by stimulus inputs (but perhaps weighted differently than the stimulus inputs to the perceived distance variable) that modulates behavior during the blind-walking task but not during the verbal report task.

It is perhaps not surprising that there is resistance to the notion that multiple pathways produce multiple internal representations of perceived distance (and the cluttered mind this implies). The existence of two distinct visual processing pathways does not necessarily require that two different representations be constructed and maintained in parallel throughout the performance of a particular task. Instead, perceptual representations (conceived of here as patterns of neural activation, not as abstract symbols) could be fleeting and closely tied to the performance of the task at hand, whether it is verbal estimation, matching of extents, blind-walking, or another behavior. In this view, perception is dynamic and plastic. Activation in the different pathways ebbs and flows - pushed and pulled by the current behavior-creating the momentary perception. Thus, perception is the product of a dynamic system involving both sensory input from the outside and the behavioral state of the perceiver "inside," in different states of interaction with the world, which, together, generate a unique pattern of neural activation and a unique representation.

The present study seeks to contribute to the two visual pathways debate and to investigate this proposal by manipulating the behavior required and the visual information available to participants in an egocentric distance perception study. We note that Philbeck and Loomis (1997) used target distances of less than $5 \mathrm{~m}$, which may have been too short for dissociation between blindwalking performance and verbal reports to emerge, given the expected accuracy of both estimates at shorter distances. In Experiment 1, we measure baseline performance for target distances from $5 \mathrm{ft}(1.52 \mathrm{~m})$ to $90 \mathrm{ft}(27.43 \mathrm{~m})$ on two different tasks: blind-walking to a previously viewed target and a verbal report of perceived distance to the target. In Experiment 2, we compare performance on each task in an indoor versus outdoor setting (with different complexes of spatial information), and in Experiment 3, we manipulate the position of the visual information on the retina, and thus its perceived relation to the body, by requiring participants to view the scene through base-up prisms, base-down prisms, or without prisms. We expect verbal estimates to reflect the oft-noted compression of perceived distance (Cutting \& Vishton, 1995; Philbeck, 2000; Sedgwick, 1986; Toye, 1986; Wagner, 1985) and to be markedly less accurate than blind-walking, which is usually remarkably precise (Loomis et al., 1992; Thomson, 1983). Philbeck and Loomis rightly argued that this difference is not in itself evidence of two distinct representations, one supporting the verbal task (formed in the ventral stream) and one supporting the action task (formed in the dorsal stream). The same result could occur if there were a single, underlying representation of egocentric distance, and performance of one of the two tasks introduced a constant transformation. Support for separate visual pathways and distinct representations would come from evidence that blind-walking and verbal reports reveal apparently different percepts and that these percepts can be independently influenced by other factors, especially by factors that there is reason to expect will have a greater impact on one stream than on the other. Thus, the relevant predictions concern interactions between type of task and other variables, in addition to a main effect of task. In our experiments we predict such effects.

\section{EXPERIMENT 1}

The goal of Experiment 1 was to obtain baseline performance measures for verbal reports and blind-walking over target distances up to $90 \mathrm{ft}$. We expected to replicate previous findings that verbal estimates of distance fall short, especially over longer distances, whereas blind-walking estimates are more accurate. 


\section{Method}

Participants. Twenty-nine university students (19 females; mean age $=20.3$ years, range $=18-22$ years) participated in Experiment 1. All of the participants had normal or corrected-to-normal vision, with no self-reported visual pathologies, and received class credit for participating.

Materials. The experiment was conducted in a gymnasium slightly larger than two side-by-side basketball courts. A golfcourse-like pattern of target distance extents was discretely mapped onto the gym floor (see Figure 1 for an example): The target location on one trial became the observation point for the next trial. Walking directions were always diagonal to the line markings on the basketball courts. The target was a 1-ft-high orange sports cone. Vision was occluded using modified flip-up welder's goggles $\left(90^{\circ}\right.$ horizontal $\times$ $42^{\circ}$ vertical approximate field of view).

Design and Procedure. After giving informed consent, the participants practiced blind-walking at a normal walking pace. This allowed the participants to get accustomed to walking while blindfolded, but they neither completed a practice trial nor received any feedback about their walking performance. A 2 (response type: verbal report or blind-walk) $\times 8$ (target distance: $5,10,15,20,30,45$, 60 , and $90 \mathrm{ft}$ ) repeated measures factorial design was used. Each distance was used twice, for a total of 16 trials, and the order of distances was randomized. Each trial began with the participant viewing the target for approximately $5 \mathrm{sec}$ and then making a verbal report of distance in feet (U.S. feet were used because of the participants' familiarity with that unit of distance). While viewing the target, the participants wore the goggles, but they had full freedom with regard to head and eye movements. After they viewed the target, their vision was occluded by lowering the visor, and they immediately began blind-walking toward the same target, stopping when they believed they had reached it. They received no feedback about their performance on individual trials. In all cases, the verbal report preceded the blind-walk. This design was necessary to prevent the participants from counting steps during the walk and then using the step count as a basis for the verbal estimate of distance. An experimenter walked quietly behind the participant, for safety. The total distance walked was measured while the participant was positioned for the next trial. The participants' vision was occluded at all times other than when they were making verbal estimates of target distances. Following the final trial, each participant was debriefed and thanked for his or her time.

\section{Results and Discussion}

Before beginning the analysis, averages of the two verbal estimates and of the two blind-walking estimates for each target distance were calculated for each participant. These averages were then divided by the target distance to get a percent accuracy score. A score greater than 1.0 represented walking (or verbal report) farther than the target distance, whereas a score less than 1.0 indicated that the participant walked (or verbally reported) a distance shorter than the target distance. A 2 (response type) $\times 8$ (distance) repeated measures ANOVA was then conducted on the percent accuracy data. Greenhouse-Geisser corrected results and effect sizes (partial eta squared, $\eta_{\mathrm{p}}^{2}$ ) are reported here.

Figure 2 depicts accuracy of distance estimates as a function of target distance for each response type. The significant main effect of response type is apparent $\left[F(1,28)=16.807, p<.001 ; \eta_{\mathrm{p}}^{2}=.375\right]$. Participants' verbal responses were less accurate $(M=78.0 \%)$ than their blind-walking estimates of distance $(M=94.6 \%)$. The main effect of target distance barely reached significance $\left[F(3.3,91.5)=2.632, p=.05 ; \eta_{\mathrm{p}}^{2}=.086\right]$, and the response type $\times$ target distance interaction was not significant $\left[F(3,85)=2.050, p=.11 ; \eta_{\mathrm{p}}^{2}=.068\right]$. Both of these factors had very small effect sizes when compared with the response type effect size.

Our findings are compatible with those reported by Philbeck and Loomis (1997, Experiment 1): Verbal estimates of perceived distance are significantly shorter than blind-walking estimates over a 5- to $90-\mathrm{ft}$ range.

\section{EXPERIMENT 2}

In Experiment 2, we investigated the influence of an indoor versus outdoor setting on verbal report and blindwalking estimates of egocentric distance. Indoor scenes are carpentered, cluttered, and limited in extent. Outdoor scenes in uncluttered open spaces may provide less ground plane structure and fewer "anchors" to help participants make distance estimates. (See Cutting \& Vishton, 1995, and Toye, 1986, for an account of how distance judgments in cluttered environments multiply constrain each other for greater veridicality of perception.) We suggest that

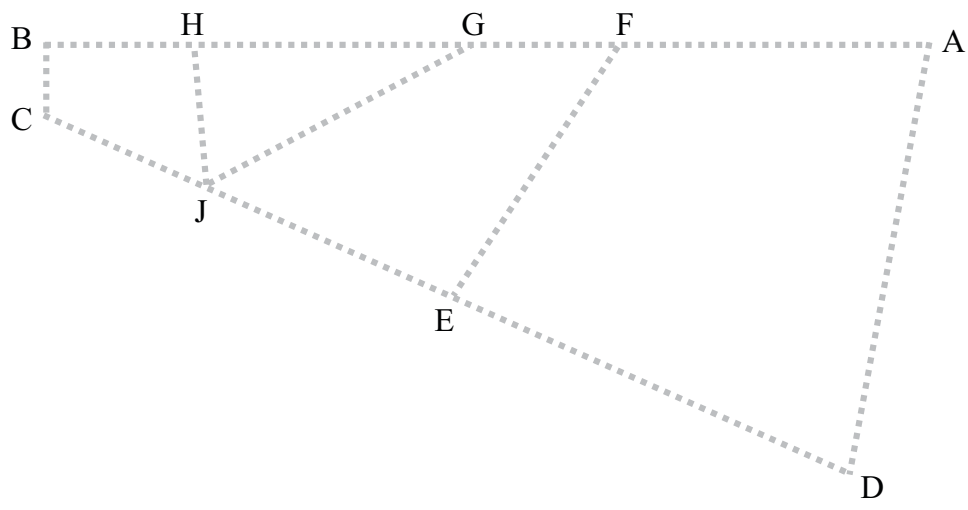

Figure 1. The "golf course" design of the walking paths. A possible path order could be line segments AB (60 ft), BC (5 ft), CE (30 ft), EF (20 ft), and FG (10 ft). 


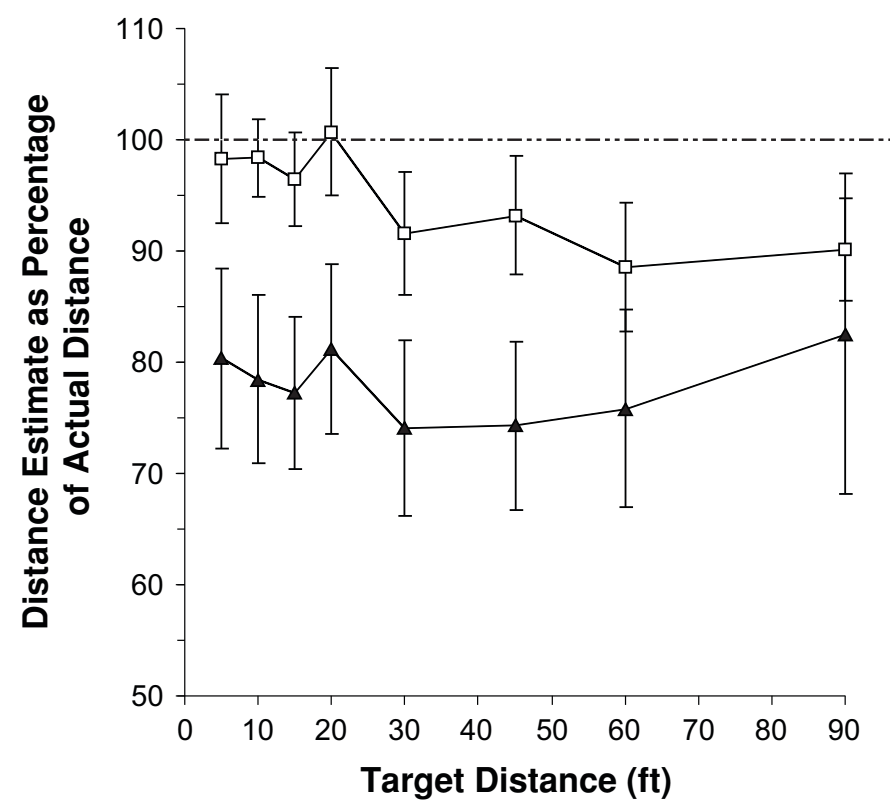

Figure 2. Experiment 1: Distance estimates as percentages of actual distance, as a function of response type at each target distance. Open symbols represent walking performance and closed symbols represent verbal reports of distance. Error bars represent $95 \%$ confidence intervals.

such information (or the lack of it) is more likely to influence a ventral stream of processing. When asked to make a verbal estimate of distance, participants are likely to attempt, quite consciously, to seek out useful objects (doors, handrails, basketball hoops, etc.) to use as metrics, and perhaps to recall known facts about intentionally humanscaled spaces. If verbal estimates require the conscious awareness of ventral-stream processing, and if a richer ventral-stream representation can be built in an indoor environment, then we would expect more accurate verbal estimates of distance in an indoor environment than in an outdoor one.

We expect less of an effect of environment on the blindwalking task. Blind-walking is expected to be only minimally affected by the contents of conscious perceptual awareness, and then only if the task requires sharing of information between the two streams. Dorsal-stream processing is likely to depend more on body-centered optical information, such as the various horizon relations based on the eye height of the observer and the visual angles subtended by extents in the scene (Rogers, 2000; Sedgwick, 1973). These variables are not expected to be affected by the indoor/outdoor environment when both include a flat, level ground plane. If the blind-walking task depends on dorsal-stream processing, and if dorsal-stream processing depends principally on body-relative optical information available in both conditions, then we would expect blindwalking to be similarly accurate in both conditions. We do not mean to imply that there is no sharing of information between streams, or that the dorsal stream cannot make use of additional optical information in the more carpentered environment in performance of the walking task. Rather, we expect that the manipulation of the environmental context will favor the ventral stream of visual processing more than the dorsal stream. Thus, we predict an interaction in which the distance estimates made using the verbal task, but not those made using the action task, will be influenced by the environmental setting. In particular, the indoor environment is expected to lead to more accurate verbal estimates of distance to the target.

\section{Method}

Participants. Seventeen university students (11 females; mean age $=20.25$ years, range $=19-22$ years) participated in Experiment 2. All of the participants had normal or corrected-to-normal vision, with no self-reported visual pathologies, and received class credit for participating.

Materials. The experiment was conducted indoors, in a gymnasium slightly larger than a basketball court, and outdoors, on a large, flat athletic training field covered with Astroturf. As in the previous experiment, a golf-course-like pattern of target distances was discretely mapped onto the gym floor and field surface; the same pattern was used both indoors and outdoors. Walking directions were always diagonal to the line markings on the gym floor and field. Target distances of 5, 10, 15, 20, 30, and $60 \mathrm{ft}(1.52-18.29 \mathrm{~m})$ were used. The target and occluding goggles were the same as in Experiment 1.

Design and Procedure. After giving informed consent, the participants practiced blind-walking; no feedback was given to the participants about their performance. A 2 (response type: verbal or blind-walking) $\times 2$ (environment: indoors or outdoors) $\times 6$ (target distance: $5,10,15,20,30$, and $60 \mathrm{ft}$ ) repeated measures factorial design was used. (The 90 -ft target distance was not used because the indoor testing environment could not accommodate it.) Each target distance occurred twice, for a total of 12 trials indoors and 
12 trials outdoors. The order of testing environments was counterbalanced across participants, and distance orders were randomized. The procedure for an individual trial was identical to the procedure in Experiment 1.

\section{Results and Discussion}

Averages of the two verbal and two blind-walking distance estimates made at each distance were calculated for each participant. These averages were again divided by the target distance to get a percent accuracy score. Estimates from one particular participant were over three standard deviations away from the mean, so this participant's data were removed from the subsequent analysis (new $N=$ 16). A 2 (response type) $\times 2$ (environment) $\times 6$ (target distance) repeated measures ANOVA was run on the percent accuracy data. Greenhouse-Geisser corrected results and effect sizes are reported below.

For comparison with the results from Experiment 1, Figure 3 illustrates the interaction between response type and environment as a function of target distance. The main effect of response type was again significant $[F(1,15)=$ $\left.14.382, p=.002 ; \eta_{\mathrm{p}}^{2}=.489\right]$ with blind-walking estimates of distance being more accurate $(M=94.5 \%$ vs. $M=$ $75.4 \%$ ). Also apparent in Figure 3 is the significant interaction between response type and environment $[F(1,15)=$ $\left.8.857, p=.009 ; \eta_{\mathrm{p}}^{2}=.371\right]$. No other main effect or interactions were significant. As predicted, only the verbal reports were influenced by the environmental setting. Verbal reports made outdoors were shorter than those made indoors, probably because the space was more open, less carpentered, and there were fewer distance anchors available for the participants to use in making the verbal estimates.

We conclude that the evidence that environmental setting has a different effect on verbal report accuracy than it does on blind-walking accuracy indicates the independence of these two tasks and provides support for the proposal that different representations result from the activation in the different visual pathways (i.e., different representations). Pathway activation, in turn, depends on the task being performed.

\section{EXPERIMENT 3}

In Experiment 3, we investigated the influence of viewing the scene through base-up prisms, base-down prisms, and without prisms on estimates of egocentric distance made through verbal report and blind-walking. Grutzmacher, Andre, and Owens (1997) reported that individuals blind-walked farther toward a target after viewing it through 10-diopter base-down prisms. They also reported that individuals walked shorter distances when the prisms were base up. They attributed these results to the optical displacement or vertical shift of the visual field when viewing through the prisms. An upward shifting of objects in the visual field is usually the result of moving them farther away, so long as they have remained on the ground plane, and so long as the eyes have not pitched up or down,

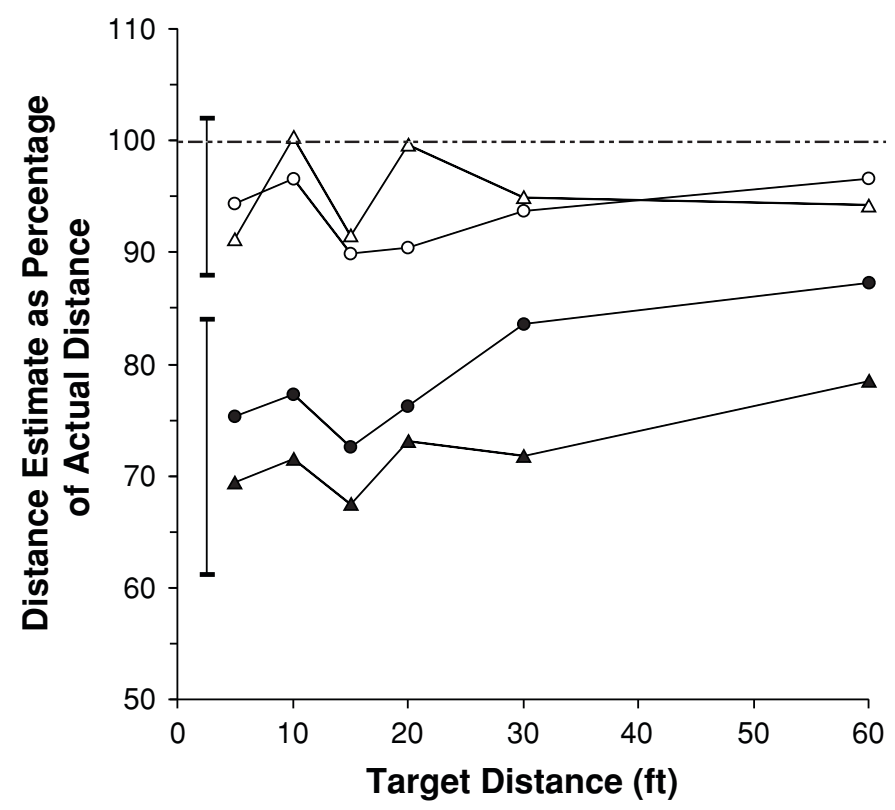

Figure 3. Experiment 2: Distance estimates as percentages of actual distance, as a function of response type and environment at each target distance. The indoor condition is depicted with circles and the outdoor condition is represented by triangles. Open symbols represent walking performance and closed symbols represent verbal reports of distance. For clarity, an average $\mathbf{9 5 \%}$ confidence interval error bar for all walking and verbal trials, respectively, has been added directly to the left of these functions. 
and so long as the observer's eye height has not changed. Thus, for a given oculomotor state and a given eye height, vertical displacement of a ground feature on the retina will result in a change in its perceived distance.

In the case of prism displacement, of course, the whole array shifts relative to the retina, not just the target object, and thus all visual angles subtended by extents within the scene, and relations among them, are preserved. Strictly in terms of the image itself, the information for distance is also preserved, adaptation to the displaced image can occur, and the effect of a change in perceived distance is likely to be short-lived. Until adaptation occurs, it is the shift in location of the image relative to the observer's body that leads to the change in the apparent distance to the target object. Horizon relations defined relative to the horizontal plane extending from the observer's eyes (instead of relative to the optical horizon specified by the perspective structure of the image) will have changed. If this body-centered information governs the representation of egocentric distance, then the apparent distance to the target will also have changed. If this representation is formed in the visuomotor dorsal processing stream, and if representations in this stream control walking to the target, then we would expect this manipulation to influence the action task of blind-walking. It is certainly possible that cognitive factors (inferences about height in the field) could influence verbal reports, but if the manipulation has no effect on the verbal reports, we would infer that there are separate underlying representations with limited sharing of information between streams. Conversely, if the prism effect reported by Grutzmacher et al. (1997) transfers to verbal reports of distance in addition to blind-walked estimates, results might be interpreted as favoring a single underlying representation of egocentric distance, but actually would not discriminate between the two options.

\section{Method}

Participants. Thirty-one university students drawn from the same subject pool population as before (age range $=18-25$ years) participated in Experiment 3. All of the participants had normal or corrected-to-normal vision, with no self-reported visual pathologies, and received class credit for participating. Because participants were required to wear prism glasses, only emmetropic individuals or contact lens wearers were able to participate.

Materials. The experiment was conducted in a large indoor gymnasium similar to the one used in Experiment 2. An identical set of walking paths, target cone, and occluding goggles were used. The participants were able to wear the 10-diopter prism glasses beneath the goggles.

Design and Procedure. After giving informed consent, the participants practiced blind-walking at a normal walking pace, but received no feedback about their performance. The participants were then tested in a 2 (response type: verbal or blind-walking) $\times$ 3 (prism: base up, none, base down) $\times 5$ (target distance: $5,10,20$, 30 , and $60 \mathrm{ft}$ ) repeated measures factorial design. Each prism $\times$ distance condition was tested twice, for a total of 30 trials. Prism condition was counterbalanced across participants, and distance orders were randomized. As in previous experiments, the participants received no feedback about their performance on any of the trials. This, coupled with the fact that the participants only viewed the environment through the prisms for very brief amounts of time, indicates that no adaptation to the prisms would have occurred during our experiment. The procedure for an individual trial was identical to the procedure in previous experiments.

\section{Results and Discussion}

Averages of the two verbal and the two blind-walking distance estimates made at each target distance were calculated for each participant in each prism condition. These averages were again divided by the target distance to get a percent accuracy score. A 2 (response type) $\times 3$ (prism) $\times 5$ (distance) repeated measures ANOVA was run on the percent accuracy data. Greenhouse-Geisser corrected results and effect sizes are reported below.

For comparison with the results from the two previous experiments, Figure 4 illustrates response type $\times$ prism condition interaction as a function of target distance. The ANOVA confirmed significant main effects of response type $\left[F(1,30)=30.632, p<.001 ; \eta_{\mathrm{p}}^{2}=.505\right]$ and prisms $\left[F(1.9,56.4)=11.105, p<.001 ; \eta_{\mathrm{p}}^{2}=.270\right]$, along with the significant interactions between response type $\times$ target distance $\left[F(2,61.3)=4.281, p=.018 ; \eta_{\mathrm{p}}^{2}=.125\right]$ and response type $\times$ prism condition $[F(1.8,55.6)=10.090, p<$ $\left..001 ; \eta_{\mathrm{p}}^{2}=.252\right]$. Once again, blind-walking estimates ( $M=99.0 \%$ of actual distance) were more accurate than verbal reports $(M=76.3 \%)$. The response type $\times$ target distance interaction, while significant, was likely only due to the increase in accuracy at 10 and $20 \mathrm{ft}$ in the walking condition. As predicted, the significant interaction between response type and prism condition shows that the performance in the base-down prism condition was only different from the no-prism condition during blind-walking (pairwise comparison post hoc test with a Bonferroni adjustment, $p<.001)$. This effect, however, was limited to the blind-walking measure-base-down prisms had no effect on verbal reports of distance $(p=.279)$. The effects of the base-up prisms were less clear, and conclusions from this prism condition cannot be made.

As in Grutzmacher et al. (1997), our participants walked farther in the base-down condition. Interestingly, however, our results differ from those of another study involving prisms. Held (1970) reported that an "ambient" or action-based task, such as eye-hand coordination, improves (adapts) with prolonged exposure to prismatic optical rearrangement. This difference is likely due to the intentional absence of adaptation in our study. Observers only briefly glimpsed the visual scene while wearing prisms.

We conclude that the evidence that prism viewing has a different effect on blind-walking accuracy than it does on verbal report accuracy indicates the independence of these two tasks and provides support for the proposal that different representations result from activation in the different visual pathways. Pathway activation, in turn, depends on the task being performed.

\section{GENERAL DISCUSSION}

We conclude that the present investigation supports the existence of two visual pathways, each of which generates a different representation of egocentric distance. Our methodology does not necessarily exclude the possibility 


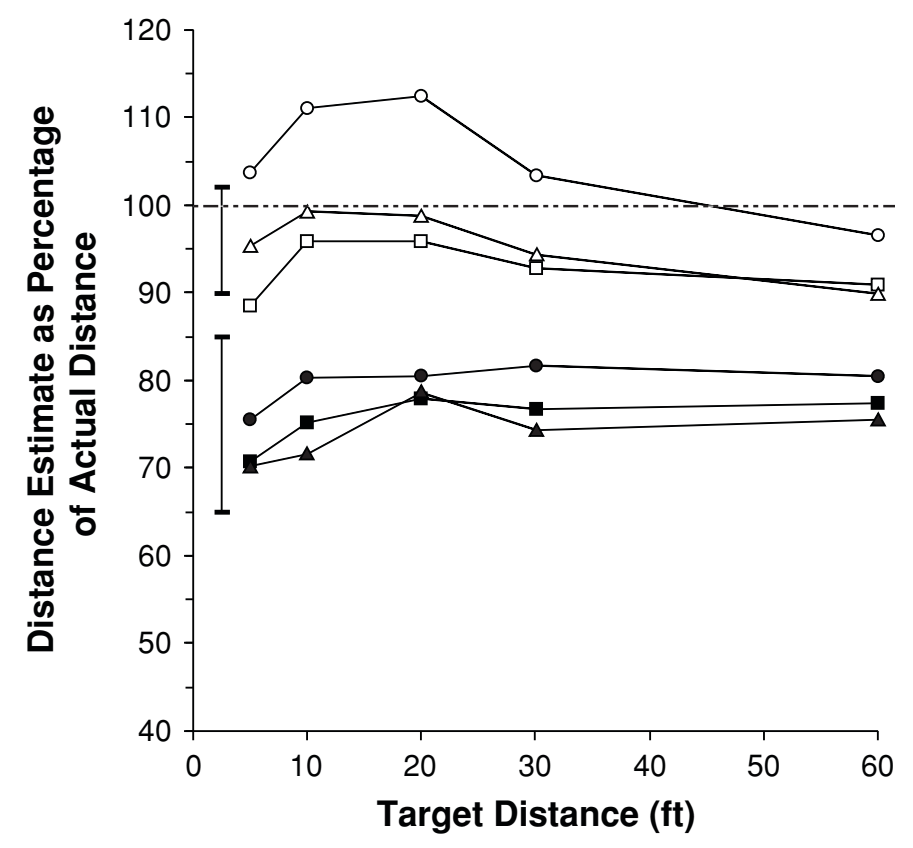

Figure 4. Experiment 3: Distance estimates as percentages of actual distance, as a function of response type and prism condition at each target distance. The base-down prism condition is depicted with circles; the no-prism condition with squares; and the base-up prism condition with triangles. Open symbols represent walking performance and closed symbols represent verbal reports of distance. For clarity, an average $95 \%$ confidence interval error bar for all walking and verbal trials, respectively, has been added directly to the left of these functions.

of two different representations being formed in the same pathway, or the possibility that there is a single representation with two output transformations influenced differently by the experimental manipulations. The evidence does not favor these alternative hypotheses, however. It is notable that the verbal task, or more precisely, either the underlying representation or the task-associated output transformation, was influenced more by a manipulation (the availability of cues to distance) expected to have an impact on the ventral stream. Similarly, the walking task and its underlying representation or its task-associated output transformation were influenced more by a manipulation (position of the visual array on the retina) that was expected to have an impact on the dorsal stream.

In Experiment 1, we established that verbal reports are indeed less accurate than blind-walked estimates of distance, and we replicated this result in Experiments 2 and 3. These results agree with those of other researchers, such as Philbeck and Loomis (1997) and Leibowitz, Guzy, Peterson, and Blake (1993), who showed that nonverbal estimates of size, distance, and duration were more accurate than their verbal counterparts. When taken together, the results from the present experiments show that the better accuracy of blind-walking is a robust, reliable finding (see Figure 5). Similar to Foley (1977), we also found that the action task showed less overall variability than the verbal reports of distance in all three experiments. This difference was statistically significant in Ex- periments 1 [paired-sample $t(28)=2.14, p=.04$ ] and 2 $[t(15)=2.59, p=.02]$. Taken together, our results bring into question the accuracy and reliability of verbal reports when estimating distance (see Leibowitz et al., 1993).

One possible explanation for the inaccuracy of verbal reporting may be that participants do not accurately know the size of a U.S. foot, the unit of measurement in which they reported their verbal estimates (R. Welch, personal communication, but see also Foley, Ribeiro-Filho, \& Da Silva (2004), who find a similar underestimation with metric units). If participants underestimate a U.S. foot, and use it as a standard for verbal estimates of distance, then one of two possibilities could occur. The participants could simply "scale up" their foot estimate to the target distance. This should lead to a proportional underestimation of target distance, and there should be a positive correlation between foot estimates and target distance estimates across all participants: smaller foot estimate, smaller reported distances. The other possibility is that the participants ask themselves "how many (underestimated) foot units could fit between me and the target?" In this case, their target distance estimates should be greater than actual distances, and correlations between foot estimates and target distance estimates should be negative: smaller foot estimates, larger number of total foot units reported between the participant and the target.

A follow-up experiment with 27 participants tested these hypotheses. Although the mean estimate of a U.S. 


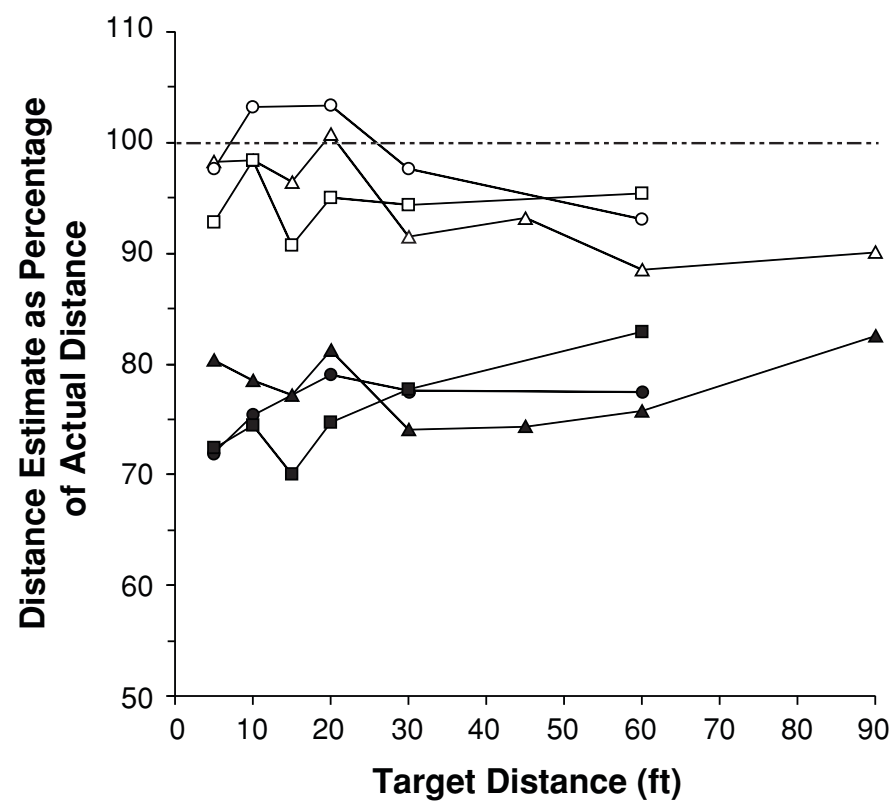

Figure 5. Overview: Distance estimates as percentages of actual distance, as a function of response type at each target distance for all three experiments. Experiment 1 is depicted with triangles, Experiment 2 with squares, and Experiment 3 with circles. Open symbols represent walking performance and closed symbols represent verbal reports of distance.

foot was approximately $79.0 \%$ (9.5 in) of the actual length of a foot, and was similar to the mean verbal estimate of the target distance ( $80.1 \%$ of the actual distance), the correlation between participants' foot estimates and mean verbal estimates of distance was not significantly different from zero $(r=-.014, p=.946)$. Correlation coefficients between participants' foot estimates and the verbal estimates for individual target distances ranged from -.256 to .065 , and none were significant. We conclude from these results that an inaccurate understanding of the length of a foot was not the basis of the inaccurate verbal reports of egocentric distance.

Most important, in Experiments 2 and 3, we demonstrated the independence of blind-walking from the verbal report task, strongly suggesting that they draw on different underlying representations of egocentric distance, generated by two different processing streams.

The outdoor environment in Experiment 2 led to a loss of accuracy and pronounced underestimates in verbal reports of distance. If both verbal and walking measures were accessing the same underlying representation of egocentric distance, we would expect walking performance to be similarly affected by the environmental context. This was not the case, because only verbal reports were affected, as predicted.

In Experiment 3, we found that viewing the scene through prisms affected walking performance but not verbal reports. As in Grutzmacher et al. (1997), individuals walked farther in the base-down prism condition than in the other conditions. Again, if both verbal and walking mea- sures were accessing the same underlying representation, we would expect both tasks to be affected by the prisms.

Our conclusion, that different representations underlie the performance of the two tasks, is at odds with the conclusion of Philbeck and Loomis (1997). In their experiment, comparing full- and reduced-cue viewing conditions, the reduction of information in the latter affected both tasks equally over their shorter range of distances. It is not possible to say whether the absence of an interaction in their data was due to the specific experimental manipulation (remove enough information and it is surely possible to underspecify distance for both putative processing streams) or to the shorter range of distances (within which, both verbal estimates and blind-walking tend to be reasonably accurate). See Cutting and Vishton (1995) for an account of the activity of various cues and sources of information within personal space, action space, and vista space. In the present investigation, we demonstrated the differential impact of two variables on the two tasks. Furthermore, the direction of the impact of the variables was consistent with the two visual systems hypothesis: The indoor environment was expected to provide additional support for the more conscious perceptuo-cognitive process supporting a verbal estimate of distance, whereas the prism displacement was expected to more strongly influence the blind-walking task.

It is important to remember that evidence that different processing streams generate different representations does not imply that two representations are constantly generated and constantly updated or that some executive 
process must choose between the two options in order for a representation to happen. If the different processing streams can be shown to generate representations differing in veridicality and utility, we can only conclude that such streams exist. It may be that no general-purpose scene description is built into either stream, nor is one assembled out of the outputs of the two streams. We suggest instead that perception is dynamic and in flux. Our apprehension of the world is driven at any moment by the task in which we are engaged. Our perceptual systems attend to the information needed to perform the task at hand, and the information is processed with the appropriate "hardware." If our task is to act on the environment, no ventralstream representation need ever be generated or enter our awareness. On the occasions in which an experimental psychologist demands a verbal report of egocentric distance, we can oblige, and enter into conversation about the appearance of the world. But our embarrassingly underestimated reports of distance will not prevent us from acting successfully.

In summary, we have reported evidence suggesting a distinction between ventral and dorsal visual processing pathways. Furthermore, we believe that these pathways generate different representations of egocentric distance, and that the representation underlying our action-based walking measure is more accurate.

\section{REFERENCES}

Creem, S. H., \& Proffitt, D. R. (2001). Defining the cortical visual systems: "What," "where," and "how." Acta Psychologica, 107, 43-68.

Cutting, J. E., \& Vishton, P. M. (1995). Information potency and spatial layout. In W. Epstein and S. J. Rogers (Eds.), Handbook of perception and cognition: Vol. 5. Perception of space and motion (2nd ed., pp. 69-117). San Diego: Academic Press.

FolEy, J. M. (1977). Effect of distance information and range on two indices of visual perceived distance. Perception, 6, 449-460.

Foley, J. M., Ribeiro-Filho, N. P., \& Da Silva, J. A. (2004). Visual perception of extent and the geometry of visual space. Vision Research, 44, 147-156.

Goodale, M. A., \& Milner, A. D. (1992). Separate visual pathways for perception and action. Trends in Neurosciences, 15, 20-25.

Grutzmacher, R. P., Andre, J. T., \& Owens, D. A. (1997). Gaze inclination: A source of oculomotor information for distance perception. In M. A. Schmuckler \& J. M. Kennedy (Eds.), Studies in perception and action $I V$ (pp. 229-232). Mahwah, NJ: Erlbaum.

Held, R. (1968). Dissociation of visual functions by deprivation and rearrangement. Psychologische Forschung, 31, 338-348.
HeLd, R. (1970). Two modes of processing spatially distributed visual stimulation. In F. O. Schmitt (Ed.), The neurosciences: Second study program (pp. 317-324). New York: Rockefeller University Press.

INGLE, D. (1967). Two visual mechanisms underlying the behavior of fish. Psychologische Forschung, 31, 44-51.

Kelly, J. W., Loomis, J. M., \& Beall, A. C. (2004). Judgments of exocentric direction in large-scale space. Perception, 33, 443-454.

Leibowitz, H. W., Guzy, L. T., Peterson, E., \& Blake, P. T. (1993). Quantitative perceptual estimates: Verbal versus nonverbal retrieval techniques. Perception, 22, 1051-1060.

Leibowitz, H. W., \& Post, R. B. (1982). The two modes of processing concept and some implications. In J. Beck (Ed.), Organization and representation in perception (pp. 343-363). Hillsdale, NJ: Erlbaum.

Loomis, J. M., Da Silva, J. A., Fujita, N., \& Fukusima, S. S. (1992). Visual space perception and visually directed action. Journal of Experimental Psychology: Human Perception \& Performance, 18, 906-921.

Loomis, J. M., Klatzky, R. L., Philbeck, J. W., \& Golledge, R. G. (1998). Assessing auditory distance perception using perceptually directed action. Perception \& Psychophysics, 60, 966-980.

Milner, A. D., \& Goodale, M. A. (1995). The visual brain in action. Oxford: Oxford University Press.

Philbeck, J. W. (2000). Visually directed walking to briefly glimpsed targets is not biased toward fixation location. Perception, 29, 259-272.

Philbeck, J. W., \& Loomis, J. M. (1997). Comparison of two indicators of perceived egocentric distance under full-cue and reduced-cue conditions. Journal of Experimental Psychology: Human Perception \& Performance, 23, 72-85.

Rogers, S. (2000). The emerging concept of information. Ecological Psychology, 12, 335-343.

SCHNEIDER, G. E. (1967). Contrasting visuomotor functions of tectum and cortex in the golden hamster. Psychologische Forschung, 31, 52-62.

SEDGWICK, H. A. (1973). The visible horizon: A potential source of visual information for the perception of size and distance. Dissertation Abstracts International, 34, 1301-1302. (UMI No. 73-22530).

SEdGwick, H. A. (1986). Space perception. In K. R. Boff, L. Kaufman, \& J. P. Thomas (Eds.), Handbook of perception and human performance: Vol. 1. Sensory processes and perception (pp. 21.1-21.57). New York: Wiley.

Thomson, J. A. (1983). Is continuous visual monitoring necessary in visually guided locomotion? Journal of Experimental Psychology: Human Perception \& Performance, 9, 427-443.

ToYE, R. C. (1986). The effect of viewing position on the perceived layout of space. Perception \& Psychophysics, 40, 85-92.

Trevarthen, C. B. (1968). Two mechanisms of vision in primates. Psychologische Forschung, 31, 299-337.

Ungerleider, L. G., \& MishKin, M. (1982). Two cortical visual systems. In D. J. Ingle, M. A. Goodale, \& R. J. W. Mansfield (Eds.), Analysis of visual behavior (pp. 549-586). Cambridge, MA: MIT Press.

Wagner, M. (1985). The metric of visual space. Perception \& Psychophysics, 38, 483-495.

(Manuscript received October 26, 2004; revision accepted for publication May 9, 2005. 